\title{
Severe COVID-19 outcomes - the role of physical activity
}

\section{Jean-Pierre Després (1)}

COVID-19 has been described as a syndemic of COVID-19 and chronic diseases. Obesity has been identified as a contributing factor to morbidity and mortality associated with COVID-19; however, sedentary behaviours and lack of physical activity should also be targeted by health authorities to reduce the risk of severe COVID-19 outcomes.

Refers to Sallis, R. et al. Physical inactivity is associated with a higher risk for severe COVID-19 outcomes: a study in 48440 adult patients. Br. J. Sports Med. https://doi.org/10.1136/bjsports-2021-104080 (2021).

The COVID-19 pandemic is having devastating human and economic consequences. Several studies conducted all over the world on cohorts of patients with COVID-19 have shown that the risk of being severely affected by this potentially deadly virus varies considerably. For instance, frail elderly people initially represented a large segment of patients with COVID-19 who died ${ }^{1}$. However, further analyses revealed that people with obesity and other chronic conditions, such as type 2 diabetes mellitus and cardiovascular disease (CVD), were at greater risk of severe COVID-19 and death than individuals of a normal weight without co-morbidities ${ }^{1,2}$. As a consequence, the COVID-19 pandemic has contributed to further raise awareness that obesity is a chronic condition requiring better management tools in clinical practice and is a public health issue that should be prioritized in population-based approaches ${ }^{3}$.

The worldwide progression in the prevalence of obesity is a marker of the 'evolution' of our lifestyles (the overall quality of our diet and our sedentary habits) that is not always compatible with human health ${ }^{4}$, and many of our living environments have become more obesogenic. Indeed, the physical demands of many of our jobs have substantially decreased, which has not been accompanied by a compensatory increase in physical activity during our leisure time $e^{5}$. As a consequence, it was highlighted several years ago that the worldwide prevalence of physically inactive individuals was greater than the prevalence of smokers, with a sedentary lifestyle potentially being a greater cause of mortality than smoking ${ }^{6}$.

Cohorts of patients with COVID-19 that included information on an index of adiposity (BMI) had all shown that individuals with obesity were at increased risk of severe COVID-19 outcomes ${ }^{1}$. However, much fewer data were available on the contribution of some of the upstream behavioural drivers of the obesity epidemic, such as level of leisure-time exercise or physical activity, which is an important knowledge gap. Regular physical activity reduces the risk of developing chronic diseases such as type 2 diabetes mellitus, CVD and respiratory diseases ${ }^{7}$. Furthermore, a high level of cardiorespiratory fitness, a physiological marker of a physically active lifestyle, is the best independent predictive factor for the development of adverse clinical outcomes, even after controlling for intermediate CVD risk factors, such as lipid levels, blood pressure and diabetes mellitus ${ }^{7}$. Such findings have led an American Heart Association consensus group to propose that cardiorespiratory fitness should become a vital sign in clinical practice ${ }^{7}$.

Some health-care organizations have embraced this concept and have treated physical activity and exercise as a clinically important behaviour to tackle. For instance, Kaiser Permanente (southern California) is well known for the attention that it gives to 'lifestyle medicine' as it actively promotes the Exercise is Medicine concept ${ }^{8}$. The Kaiser Permanente electronic health record includes information on the level of physical activity for inpatients and outpatients, which is systematically measured by questionnaires. In the current COVID-19 pandemic, investigators from this organization were therefore in a unique position to examine the potential benefits of being physically active on hospitalization rates, intensive care unit admissions and mortality for patients with COVID-19. This hypothesis was tested in a sample of 48,440 adult patients with a COVID-19 diagnosis ${ }^{8}$.

\section{These important findings} show how critical it is to assess level of physical activity in clinical practice

This study included patients who had a COVID-19 diagnosis between 1 January 2020 and 21 October 2020 and for whom at least three exercise measurements were available (so-called exercise vital sign, which was self-reported at three distinct visits). The participants were divided into three physical activity categories: 1 , consistently inactive (0-10 min per week); 2 , some activity but below guidelines (11-149 min per week); and 3, consistently meeting guidelines $(\geq 150 \mathrm{~min}$ per week). Patients with COVID-19 who were consistently inactive during the 2 years preceding the pandemic had 2.26 times greater odds for hospitalization (95\% CI: 1.81-2.83) than patients who were consistently meeting physical activity guidelines. Consistently inactive patients were also characterized by greater odds for admission to the intensive care unit (OR 1.73, 95\% CI 1.18-2.55) and for death (OR 2.49, 95\% CI 1.33-4.67) compared with patients who were consistently meeting guidelines. Compared with consistently inactive patients, patients with some activity (but below guideline levels) were at lower risk of hospitalization and death, consistent with the notion that some activity is better than nothing. However, these patients with suboptimal levels of physical activity were at substantially greater risk of all adverse COVID-19 outcomes than those consistently meeting physical activity recommendations.

These important findings show how critical it is to assess level of physical activity in clinical practice. Indeed, the risk of adverse COVID-19 outcomes associated with being physically inactive was higher than that 
of smoking and most chronic conditions (obesity, diabetes mellitus, hypertension, CVD and cancer) considered in the analyses. Thus, these results show the critical role of a sedentary lifestyle not only as a risk factor for chronic diseases but also for severe COVID-19 complications and death.

\section{$\int$ a little physical activity is better than nothing at all}

A few points regarding this interesting study deserve discussion. Firstly, level of exercise was self-reported by patients. Thus, although statistically significant relationships were found, there is often misclassification of individuals when using reported physical activity data ${ }^{9}$. Nevertheless, the associations were robust, suggesting that had direct measurements been obtained (for example, accelerometry data or direct measurements of cardiorespiratory fitness), even stronger associations might have been observed. Secondly, even suboptimal levels of exercise (below guidelines) were better than inactivity in terms of risk of severe COVID-19 outcomes. This is a very important and encouraging message for clinicians to convey to their patients: a little physical activity is better than nothing at all. Finally, even with reported data, only 3,118 of the 48,440 patients with COVID-19 (6.4\%) met the physical activity guidelines, highlighting the ubiquitous prevalence of sedentary lifestyles in our society.

These results show how an inexpensive and simple behaviour such as a daily $30 \mathrm{~min}$ walk 5 days per week could have a substantial effect on the risk of developing severe COVID-19 outcomes (FIG. 1). For instance, in addition to its effect on all known CVD risk factors, regular physical activity and exercise can have an influence on emerging CVD risk markers. As it has been suggested that COVID-19 is an endothelial disease ${ }^{2}$, the beneficial effects of regular physical activity and exercise on endothelial function and vascular health should be kept in mind ${ }^{2}$. Furthermore, visceral and ectopic adipose depots are important sources of inflammatory cytokines that contribute to the deleterious outcomes of COVID-19 (REF. ${ }^{1}$ ). Intervention studies and

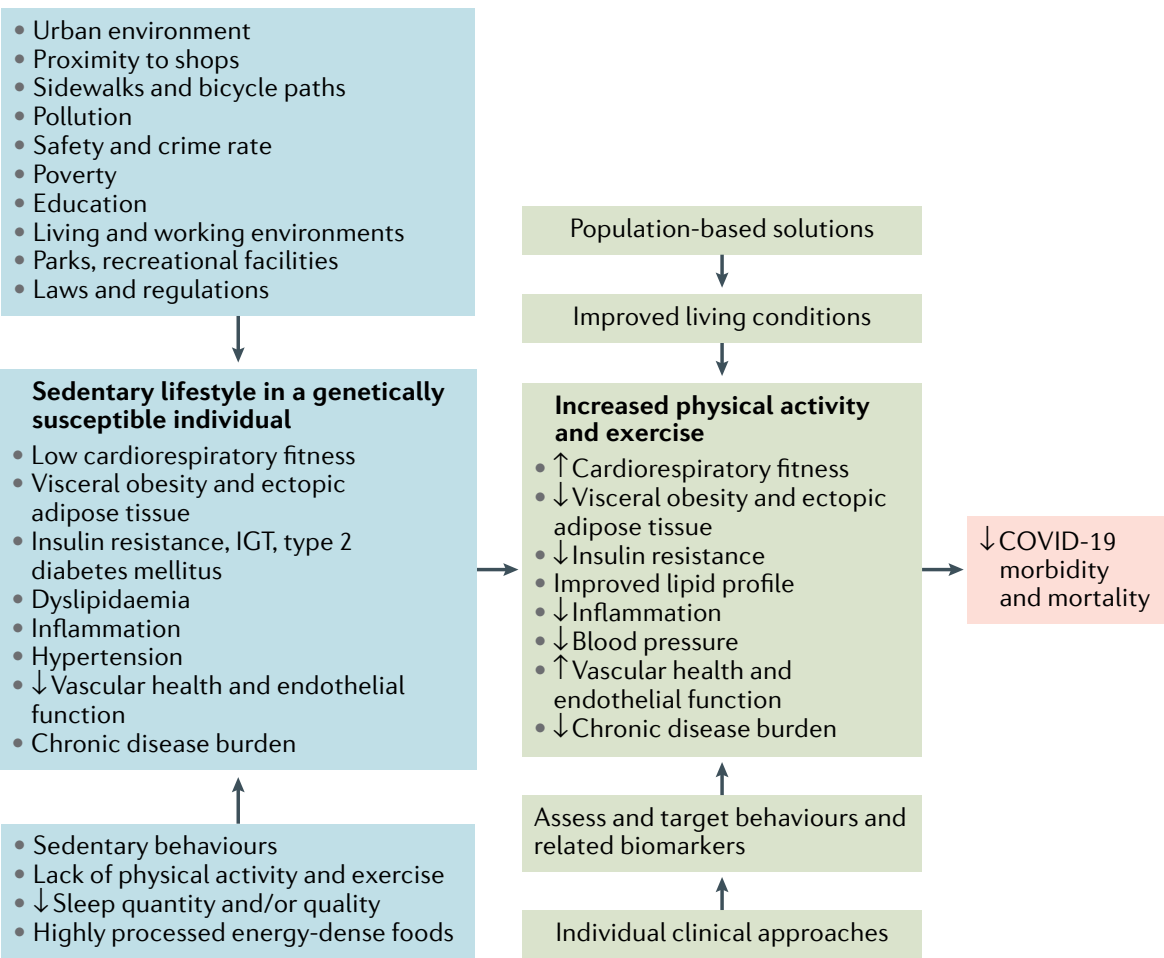

Fig. 1 | Physical activity as a key upstream target to reduce the risk of COVID-19 morbidity and mortality. Available evidence suggests that some features of disrupted metabolism, including inflammation, result from a high-risk adiposity phenotype (excess visceral adiposity or ectopic adipose tissue). As regular physical activity and exercise can reduce the size of these adipose depots, a physically active lifestyle might be a key, inexpensive, behaviour to adopt to reduce the risk of COVID-19 morbidity and mortality. However, combining population-based approaches promoting an active lifestyle with the assessment of physical activity and/or cardiorespiratory fitness and sedentary behaviours as 'vital signs' in clinical practice will be required. IGT, impaired glucose tolerance. meta-analyses have shown the effect of exercise on reducing visceral adiposity ${ }^{10}$, indicating another way in which exercise could contribute to limiting the inflammatory response to the COVID-19 infection.

In summary, more than ever, the devastating outcomes of the COVID-19 crisis have revealed that we need to provide clinicians with tools to assess and target physical activity in clinical practice. Furthermore, considering the syndemic of chronic diseases and COVID-19, we also need to reshape our living environments, including our schools and working places, so that they promote health rather than chronic diseases.

Jean-Pierre Després (iD 1,2,3

'VITAM - Centre de recherche en santé durable, CIUSSS de la Capitale-Nationale, Québec, Québec, Canada.

${ }^{2}$ Department of Kinesiology, Faculty of Medicine, Université Laval, Québec, Québec, Canada.

${ }^{3}$ Centre de recherche de l'Institut universitaire de cardiologie et de pneumologie de Québec - Université Laval, Québec, Québec, Canada.

e-mail: Jean-Pierre.Despres@criucpq.ulaval.ca https://doi.org/10.1038/s41574-021-00521-1

1. Sattar, N., McInnes, I. B. \& McMurray, J. J. V. Obesity is a risk factor for severe COVID-19 infection: multiple potential mechanisms. Circulation 142, 4-6 (2020).

2. Libby, P. \& Luscher, T. COVID-19 is, in the end, an endothelial disease. Eur. Heart J. 41, 3038-3044 (2020).

3. O'Rourke, R. W. \& Lumeng, C. N. Pathways to severe COVID-19 for people with obesity. Obesity 29 , 645-653 (2021).

4. Bluher, M. Obesity: global epidemiology and pathogenesis. Nat. Rev. Endocrinol. 15, 288-298 (2019).

5. Church, T. S. et al. Trends over 5 decades in U.S. occupation-related physical activity and their associations with obesity. PLOS ONE 6, e 19657 (2011).

6. Wen, C. P. $\&$ Wu, X. Stressing harms of physical inactivity to promote exercise. Lancet 380, 192-193 (2012).

7. Ross, R. et al. Importance of assessing cardiorespiratory fitness in clinical practice: a case for fitness as a clinical vital sign: a scientific statement from the American Heart Association. Circulation 134, e653-e699 (2016).

8. Sallis, R. et al. Physical inactivity is associated with a higher risk for severe COVID-19 outcomes: a study in 48440 adult patients. Br. J. Sports Med. https://doi.org/10.1136/bjsports-2021-104080 (2021).

9. Prince, S. A. et al. A comparison of direct versus selfreport measures for assessing physical activity in adults: a systematic review. Int. J. Behav. Nutr. Phys. Act. 5, 56 (2008).

10. Rao, S. et al. Effect of exercise and pharmacological interventions on visceral adiposity: A systematic review and meta-analysis of long-term randomized controlled trials. Mayo Clin. Proc. 94, 211-224 (2019).

\section{Acknowledgements}

J.P.D. is the Scientific Director of the International Chair on Cardiometabolic Risk supported by the Fondation de l'Université Laval. Research from J.P.D. is currently supported by the Canadian Institutes of Health Research (Foundation grant: FDN-167278) as well as by the Fondation of the Québec Heart and Lung Institute.

Competing interests

The author declares no competing interests. 